\title{
SAR Image Segmentation Using Level Sets and Region Competition under the $\mathcal{G}^{H}$ Model
}

\author{
Maria Elena Buemi, Norberto Goussies, Julio Jacobo, and Marta Mejail \\ Departamento de Computación, Facultad de Ciencias Exactas y Naturales, \\ Universidad de Buenos Aires, \\ Ciudad Universitaria, Pabellón I, 1428 Ciudad de Buenos Aires, República Argentina \\ \{mebuemi, ngoussie, jacobo, marta\}@dc.uba.ar \\ http://www-2.dc.uba.ar/grupinv/imagenes/
}

\begin{abstract}
Synthetic Aperture Radar (SAR) images are dificult to segment due to their characteristic noise, called speckle, which is multiplicative, non-gaussian and has a low signal to noise ratio. In this work we use the $\mathcal{G}^{H}$ distribution to model the SAR data from the different regions of the image. We estimate their statistical parameters and use them in a segmentation algorithm based on multiregion competition. We then apply this algorithm to segment simulated as well as real SAR images and evaluate the accuracy of the segmentation results obtained.
\end{abstract}

Keywords: SAR images, $\mathcal{G}^{H}$ distribution, multiregion competition, level set, segmentation.

\section{Introduction}

Several types of imaging devices employ coherent illumination as, for instance, Synthetic Aperture Radar (SAR), sonar, laser and ultrasound-B. The images generated by these devices are affected by a noise called speckle, a kind of degradation that does not obey the classical hypotheses of being Gaussian and additive. Speckle noise reduces the ability to extract information from the data, so specialized techniques are required to deal with such imagery. Identifying boundaries that separate different areas is one of the most important image understanding goals. High level image processing relies on precise and accurate boundaries, among other features. Finding boundaries between regions of different roughness is a hard task when data are contaminated by speckle noise. Speckled data can be statistically modeled using the family of $\mathcal{G}$ distributions [1, since these probability laws are able to describe the observed data better than other laws, specially in the case of rough and extremely rough areas. As a case of interest, in SAR images such situations are common when scanning urban spots or forests on undulated relief, and for them the more classical $\Gamma$ and $\mathcal{K}$ distributions do no exhibit good performance [12]. Under the $\mathcal{G}$ model, regions with different degrees of roughness can be characterized by the statistical parameters. Therefore, this information can be used to find boundaries between regions with different textures. The propose of this work is to use region competition methods

E. Bayro-Corrochano and J.-O. Eklundh (Eds.): CIARP 2009, LNCS 5856, pp. 153-160, 2009.

(C) Springer-Verlag Berlin Heidelberg 2009 
under $\mathcal{G}^{H}$. We replace the hypothesis of Gaussian noise in the region competition functional 3] with the hypothesis of $\mathcal{G}^{H}$ distributed noise. The minimization of the resulting functional is performed via the level set formalism 4. This work is structured as follows, in section 2 we describe the $\mathcal{G}^{H}$ distribution, in section 3 we describe the segmentation process based on region competition and level set minimization, in section 4 we present the results on simulated and real images and our conclusions.

\section{Image Model and the $\mathcal{G}^{H}$ Distribution}

Monopolarized SAR images can be modeled as the product of two independent random variables: one corresponding to the backscatter $X$, which is a physical quantity that depends on the geometry and the electromagnetic characteristics of the sensed surface, and the other one corresponding to the speckle noise $Y$, the typical noise of coherent illumination devices. In this manner

$$
Z=X \cdot Y
$$

models the return $Z$ in each pixel under the multiplicative model. For monopolarized data, the speckle noise $Y$ is modeled as a $\Gamma(n, n)$ distributed random variable, where $n$ is the number of looks, so its density is given by

$$
f_{Y}(y)=\frac{n^{n}}{2^{n} \Gamma(n)} y^{n-1} \exp \left(-\frac{1}{2} n y\right), y>0 .
$$

Also for this type of data, the backscatter $X$ is considered to obey a Generalized Inverse Gaussian law, denoted as $\mathcal{N}^{-1}(\alpha, \lambda, \gamma)$ [5]. This distribution has been proposed as a general model for backscattering, its density function being

$$
f_{X}(x)=\frac{(\lambda / \gamma)^{\alpha / 2}}{2 K_{\alpha}(\sqrt{\lambda \gamma})} x^{\alpha-1} \exp \left(-\frac{1}{2}\left(\lambda x+\frac{\gamma}{x}\right)\right), x>0 .
$$

The values of the statistical parameters $\gamma, \lambda$ and $\alpha$ are constrained to be: $\gamma>0$ and $\lambda \geq 0$ when $\alpha<0, \gamma>0$ and $\lambda>0$ when $\alpha=0$, and $\gamma \geq 0$ and $\lambda>0$ when $\alpha>0$. The function $K_{\alpha}$ is the modified Bessel function of the third kind.

The backscatter $X$ can exhibit different degrees of roughness and therefore, considering this characteristic, it could follow different models.

For smooth areas, such as pastures and many types of crops, a constant $\mathcal{C}$ distribution is an adequate model for $X$. For homogeneous and also for moderately heterogeneous areas, the $\Gamma$ distribution is a good model, and the corresponding distribution for the $\mathrm{SAR}$ data $Z$ is the $\mathcal{K}$ distribution .

In order to model a wide range of targets, ranging from rough to extremely rough targets, the reciprocal of Gamma $\Gamma^{-1}$ [1] and the Inverse Gaussian $I G(\gamma, \lambda)$ distributions can be used. This in turn results in the $\mathcal{G}^{0}$ 67829, and the $\mathcal{G}^{H}$ distributions for the return $Z$, respectively. These distributions have the additional advantage of their mathematical tractability, when compared to the $\mathcal{K}$ distribution. 
In this paper, we propose the use of the Inverse of Gamma distribution to model the backscatter $X$. This statistical law is the result of making $\alpha=-1 / 2$ in the Generalized Inverse Gaussian distribution $\mathcal{N}^{-1}(\alpha, \lambda, \gamma)$ so it becomes the $I G(\gamma, \lambda)$ distribution.

The density function of this distribution is given by (Eq. 4).

$$
f_{X}(x)=\sqrt{\frac{\gamma}{2 \pi x^{3}}} \exp \left(-\frac{(\sqrt{\lambda} x-\sqrt{\gamma})^{2}}{2 x}\right), x>0,
$$

with $\lambda, \gamma>0$. The parameters $\gamma$ and $\lambda$ can be used to define a new pair of parameters $\omega$ and $\eta$, given by $\omega=\sqrt{\gamma \lambda}, \eta=\sqrt{\gamma / \lambda}$ so formula (4) can be rewritten as

$$
f_{X}(x)=\sqrt{\frac{\omega \eta}{2 \pi x^{3}}} \exp \left(-\frac{1}{2} \omega \frac{(x-\eta)^{2}}{x \eta}\right), x>0 .
$$

So $X \sim I G(\omega, \eta)$, and it is possible to see that the corresponding moments are

$$
\mathbb{E}\left[X^{r}\right]=\sqrt{\frac{2 \omega}{\pi}} \exp (\omega) \eta^{r} K_{r-\frac{1}{2}}(\omega) .
$$

where $K_{r-\frac{1}{2}}(\omega)$ is the modified Bessel function of the third kind. Given that the order of this function is $r-\frac{1}{2}$ with $r$ an integer number, there is a closed formula that allows it to be easily evaluated.

The corresponding density function for the return $Z$, is given by

$$
\begin{aligned}
f_{\mathcal{G}^{H}}(z)=\frac{n^{n}}{\Gamma(n)} \sqrt{\frac{2 \omega \eta}{\pi}} \exp (\omega)\left(\frac{\omega}{\eta(\omega \eta+2 n z)}\right)^{n / 2+1 / 4} \\
\cdot z^{n-1} K_{n+1 / 2}\left(\sqrt{\frac{\omega}{\eta}(\omega \eta+2 n z)}\right),
\end{aligned}
$$

with $\omega, \eta, z>0$ and $n \geq 1$, respectively.

The moments of the $\mathcal{G}^{H}$ distribution are

$$
E_{\mathcal{G}^{H}}\left(Z^{r}\right)=\left(\frac{\eta}{n}\right)^{r} \exp (\omega) \sqrt{\frac{2 \omega}{\pi}} K_{r-1 / 2}(\omega) \frac{\Gamma(n+r)}{\Gamma(n)},
$$

and are used to estimate the statistical parameters.

\section{Image Segmentation}

Let $I: \Omega \rightarrow \Re$ be an image defined over $\Omega \subseteq \Re^{2}$. The goal of the segmentation process is to find a family of regions $\mathcal{R}=\left\{\mathbf{R}_{i}\right\}_{i=1 \ldots N}$ such that:

- Each region is a subset of the image domain $\mathbf{R}_{i} \subseteq \Omega$.

- The regions are pairwise disjoint $\mathbf{R}_{i} \cap \mathbf{R}_{j}=\phi \forall i \neq j$.

- Cover the image domain $\cup_{i=1}^{N} \mathbf{R}_{i} \subseteq \Omega$.

- The points in each region share some image characteristics. 
In [3] Zhu and Yuille proposed that the intensity values of the points inside each region are consistent with having been generated by one of a family of prespecified probability distributions $P\left(I(\boldsymbol{x}): \boldsymbol{\alpha}_{\boldsymbol{i}}\right)$, where $\boldsymbol{\alpha}_{\boldsymbol{i}}$ are the parameters of the distribution for the region $\mathbf{R}_{i}$. In [3] the image segmentation problem is posed as the minimization of the energy functional:

$$
E^{Z Y}\left(\mathbf{R}_{1}, \ldots, \mathbf{R}_{N}, \boldsymbol{\alpha}_{1}, \ldots, \boldsymbol{\alpha}_{\boldsymbol{N}}\right)=\sum_{i=1}^{N}\left(-\int_{\mathbf{R}_{i}} \log P\left(I(\boldsymbol{x}): \boldsymbol{\alpha}_{\boldsymbol{i}}\right) d \boldsymbol{x}+\frac{\mu}{2} \oint_{\partial \mathbf{R}_{i}} d s\right)
$$

being $\partial \mathbf{R}_{i}$ the boundary of the region $\mathbf{R}_{i}$. The regions that minimizes the functional are the desired family of regions $\mathcal{R}$. The first term, is the sum of the cost for coding the intensity of every $\boldsymbol{x}$ pixel inside the $\mathbf{R}_{i}$ according to it's distribution. The second term, is a regularization term and penalizes large boundaries. The parameter $\mu>0$ is a weighting constant controlling the regularization. In this work we assume that $I(\boldsymbol{x}) \sim \mathcal{G}^{H}$, therefore $P\left(I(\boldsymbol{x}): \boldsymbol{\alpha}_{\boldsymbol{i}}\right)$ is given by Eq. (7) and $\boldsymbol{\alpha}_{\boldsymbol{i}}=\left(\omega_{i}, \eta_{i}\right)$.

\subsection{Level Sets Based Minimization}

Although the suggested functional in Eq. (9) describes the problem quite accurately, their minimization is very difficult. Level sets based methods are a way to solve this problem. The methods has a lot of attractive properties. First, level sets can describe topological changes in the segmentation. Second, it is not necessary to discretisize the contours of the objects.

Level sets [4 based methods to minimize functionals like Eq. (9) has been addressed by multiple works 10]11/12]13. Most of them uses more than one level set function to represent the regions. The main difficulty is that the evolution of level set functions need to be coupled in order respect the restrictions of disjoint regions. In the two-region case this constraint is implicitly satisfied.

In 10. Chan and Vese extended the work in [14 to deal multiple regions using only $\log N$ level set functions. When the number of regions is a power of 2 , this model implicitly respect the restriction that the regions are disjoint. However when the number of level set functions is not a power of two this model shows some problems. The first problem is that region boundaries are weighted twice. The second problem is that the model introduces empty regions.

A different approach is proposed in 13 where $N-1$ level set functions $\left\{\Phi_{i}\right\}_{i=1 \ldots N-1}$ are used to represent $N$ regions. In the work they define the regions $R_{\Phi_{i}}=\{x \in \Omega \mid \Phi(x)>0\}$ and the desired segmentation is given by the family $\mathcal{R}=\left\{R_{\Phi_{1}}, R_{\Phi_{1}}^{c} \cap R_{\Phi_{2}}, R_{\Phi_{1}}^{c} \cap R_{\Phi_{2}}^{c} \cap R_{\Phi_{3}}, \ldots, R_{\Phi_{1}}^{c} \cap \ldots \cap R_{\Phi_{N-1}}^{c}\right\}$ which satisfies the partition constraint by definition. The proposed coupled motion equations are:

$$
\frac{\partial \Phi_{j}}{\partial t}(\boldsymbol{x}, t)=\left\|\nabla \Phi_{j}(\boldsymbol{x}, t)\right\|\left(P\left(I(\boldsymbol{x}): \alpha_{j}\right)-\psi_{j}(\boldsymbol{x})+\mu \operatorname{div}\left(\frac{\nabla \Phi_{j}(\boldsymbol{x}, t)}{\left\|\nabla \Phi_{j}(\boldsymbol{x}, t)\right\|}\right)\right)
$$

with $1 \leq j \leq N-1$, and where $\psi_{j}(\boldsymbol{x})$ is given by: 


$$
\begin{aligned}
& \psi_{j}(\boldsymbol{x})=P\left(I(\boldsymbol{x}): \alpha_{j+1}\right) \chi_{R_{\Phi_{j+1}}}(\boldsymbol{x})
\end{aligned}
$$

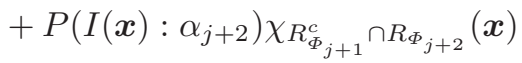

$$
\begin{aligned}
& \text {... }
\end{aligned}
$$

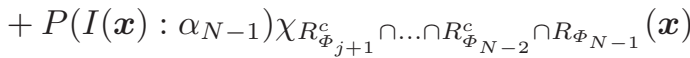

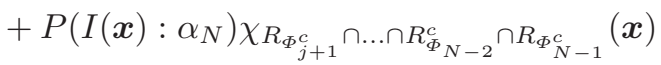

The last approach described is simple and easy to implement. It has been successfully used in SAR segmentation images in the work [15]. Thereof this is the approach we have adopted in our work.

\section{Results and Conclusions of Image Segmentation Using $G^{H}$ Models}

The proposed algorithm has been tested on a range of simulated and real images. The results for two simulated images are shown in Fig. 1 (a) through (d) and Fig. 1 (e) through (h), showing from left to right, the initial contours, their evolution and final results. These images were generated using the $\mathcal{G}^{H}$ distribution. The parameters used to generate each of the regions and their corresponding estimates from the segmented images are shown in Table 1 The percentage of pixels correctly classified in the first image is $97.05 \%$ and in the second image is $96.89 \%$. The obtained results for the segmentation of simulated images are similar in performance to those obtained by [15].

Table 1. Values for the parameters used to generate the simulated data in Fig. 1(a) and Fig. 1(e) and their corresponding estimates, calculated from the segmented regions depicted in Fig. 1(c) and Fig. 1(g)

\begin{tabular}{|l|cc|cc|cc|cc|}
\hline & \multicolumn{3}{|c|}{ Figure 1(a) } & \multicolumn{4}{c|}{ Figure 1(e) } \\
\hline Region color & $\eta$ & $\omega$ & $\eta$-estimate $\omega$-estimate & $\eta$ & $\omega$ & $\eta$-estimate $\omega$-estimate \\
\hline background & 2.75 & 57.60 & 2.47 & 55.8 & 13.4 & 7.4 & 13.62 & 7.37 \\
dark gray & 3.1 & 10.5 & 2.88 & 10.57 & 1.95 & 67.60 & 1.33 & 56.97 \\
light gray & 1.08 & 2.25 & 1.13 & 2.20 & 8.1 & 15.50 & 7.71 & 15.14 \\
white & 10.0 & 5.0 & 7.07 & 4.90 & 1.43 & 3.16 & 1.51 & 3.15 \\
\hline
\end{tabular}

Table 2. Estimated $\mathcal{G}^{H}$ parameters for the segmented regions shown in Fig. 2(c) and Fig. 2(g)

\begin{tabular}{|l|c|c|c|c|}
\hline & \multicolumn{2}{|c|}{ Figure [2(a) } & \multicolumn{2}{|c|}{ Figure[2] $(\mathrm{e})$} \\
\hline Region color & $\eta$ & $\omega$ & $\eta$ & $\omega$ \\
\hline background & 2.60 & 3.38 & 1.06 & 2.09 \\
dark gray & 12.93 & 2.95 & 3.21 & 17.46 \\
light gray & 66.85 & 3.08 & 46.54 & 0.62 \\
white & - & - & 9.14 & 2.35 \\
\hline
\end{tabular}




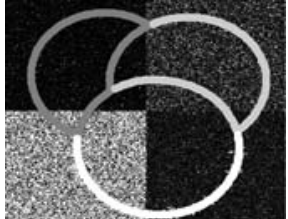

(a)

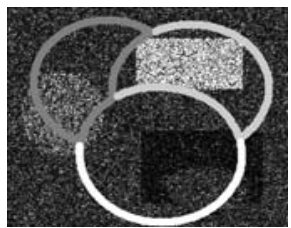

(e)

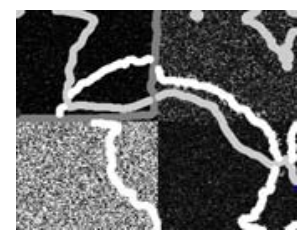

(b)

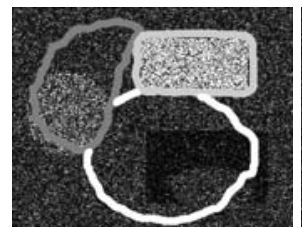

(f)

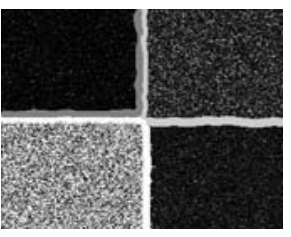

(c)

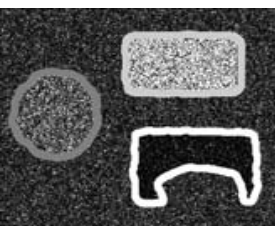

(g)

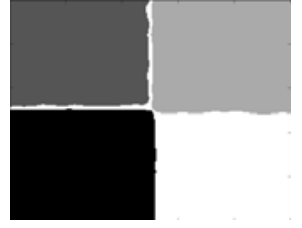

(d)

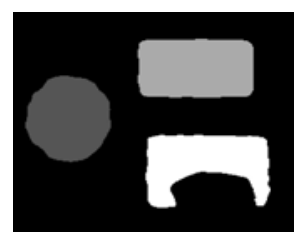

(h)

Fig. 1. Results for two simulated images. (From left to right) Column 1: initial curves, Column 2: position of curves at iteration 11, Column 3: final position of curves, Column 4: segmentation result. The segmented regions and their corresponding contours are shown with the same gray level.

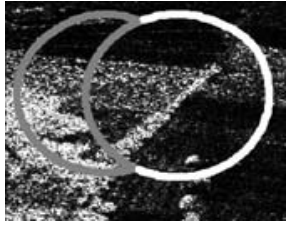

(a)

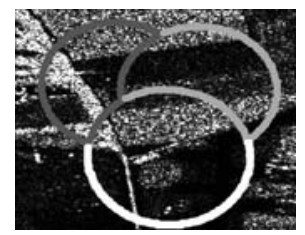

(e)

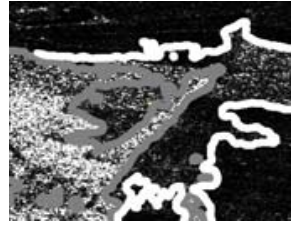

(b)

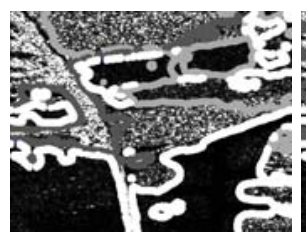

(f)

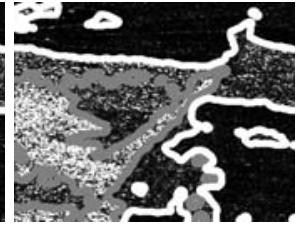

(c)

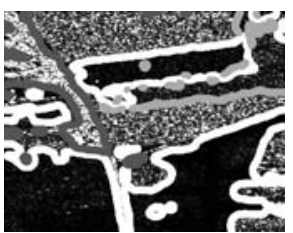

(g)

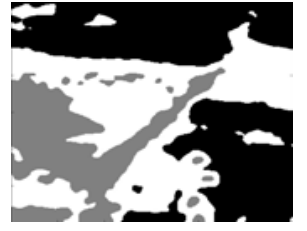

(d)

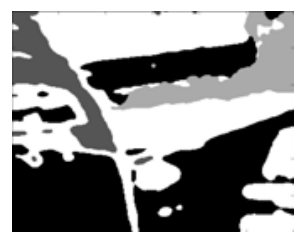

$(\mathrm{h})$

Fig. 2. Results for two real images: (From left to right), Column 1: initial curves, Column 2: position of curves at iteration 11, Column 3: final position of curves, Column 4: segmentation result. The segmented regions and their corresponding contours are shown with the same gray level.

Results for two real images with different number of regions are shown in Fig. 2 (a) through (d) and Fig. 2 (e) through (h), here again showing, from left to right, the initial contours, their evolution and final results. These real images were extracted from an E-SAR image acquired over the DLR location at Oberpfaffenhofen, Germany. The estimated parameters are shown in Table 2 . The number of regions used in the segmentation of each of the images were 
estimated by visual inspection. The results obtained on these images show good segmentation performance for the proposed method. As an example of this, we can exhibit the dark gray region in the segmentation result of the Fig. 2(e) which has a small estimated value for the statistical parameter $\omega$, meaning that there should be buildings in that region. This can be confirmed from a visual inspection of maps of this area.

The presented results support the idea that characterization of regions in SAR images through the use of statistical parameters of the $\mathcal{G}^{H}$ distribution is very useful and it can be incorporated succesfully in a level set based segmentation scheme.

\section{References}

1. Frery, A.C., Müller, H.-J., Yanasse, C.C.F., Sant'Anna, S.J.S.: A model for extremely heterogeneous clutter. IEEE Transactions on Geoscience and Remote Sensing 35(3), 648-659 (1997)

2. Mejail, M.E., Frery, A.C., Jacobo-Berlles, J., Bustos, O.H.: Approximation of distributions for SAR images: proposal, evaluation and practical consequences. Latin American Applied Research 31, 83-92 (2001)

3. Zhu, S.C., Yuille, A.: Region competition: Unifying snakes, region growing, and bayes/mdl for multiband image segmentation. IEEE Transactions on Pattern Analysis and Machine Intelligence 18, 884-900 (1996)

4. Sethian, J.A.: Level Set Methods and Fast Marching Methods: Evolving Interfaces in Computational Geometry, Fluid Mechanics, Computer Vision, and Materials Science. Cambridge University Press, Cambridge (2007)

5. Frery, A.C., Correia, A., Renno, C.D., Freitas, C.D.C., Jacobo-Berlles, J., Vasconcellos, K.L.P., Mejail, M., Sant'Anna, S.J.S.: Models for synthetic aperture radar image analysis. Resenhas (IME-USP) 4, 45-77 (1999)

6. Jacobo-Berlles, J., Mejail, M., Frery, A.C.: The ga0 distribution as the true model for sar images. In: SIBGRAPI 1999: Proceedings of the XII Brazilian Symposium on Computer Graphics and Image Processing, Washington, DC, USA, pp. 327-336. IEEE Computer Society, Los Alamitos (1999)

7. Mejail, M., Frery, A.C., Jacobo-Berlles, J., Kornblit, F.: Approximation of the ka distribution by the ga. In: Second Latinoamerican Seminar on Radar Remote Sensing: Image Processing Techniques, pp. 29-35 (1999)

8. Mejail, M.E., Jacobo-Berlles, J., Frery, A.C., Bustos, O.H.: Classification of sar images using a general and tractable multiplicative model. International Journal of Remote Sensing 24(18), 3565-3582 (2003)

9. Quartulli, M., Datcu, M.: Stochastic geometrical modelling for built-up area understanding from a single SAR intensity image with meter resolution. IEEE Transactions on Geoscience and Remote Sensing 42(9), 1996-2003 (2004)

10. Vese, L.A., Chan, T.F.: A multiphase level set framework for image segmentation using the mumford and shah model. International Journal of Computer Vision 50, 271-293 (2002)

11. Chung, G., Vese, L.: Energy minimization based segmentation and denoising using a multilayer level set approach. Energy Minimization Methods in Computer Vision and Pattern Recognition, 439-455 (2005) 
12. Brox, T., Weickert, J.: Level set segmentation with multiple regions level set segmentation with multiple regions. IEEE Transactions on Image Processing 15(10), 3213-3218 (2006)

13. Mansouri, A.R., Mitiche, A., Vazquez, C.: Multiregion competition: A level set extension of region competition to multiple region image partitioning. Computer Vision and Image Understanding 101(3), 137-150 (2006)

14. Chan, T.F., Vese, L.A.: Active contours without edges. IEEE Transactions on Image Processing 10(2), 266-277 (2001)

15. Ayed, I.B., Mitiche, A., Belhadj, Z.: Multiregion level-set partitioning of synthetic aperture radar images. IEEE Transactions on Pattern Analysis and Machine Intelligence 27(5), 793-800 (2005) 